\title{
TRATAMENTO DE FENANTRENO UTILIZANDO PERSULFATO DE SÓDIO ATIVADO POR DIATOMITA MODIFICADA POR FERRO
}

\author{
S.S.O. SILVA. ${ }^{1}$; A.R. SOUZA ${ }^{1}$; A. G. CÂMARA ${ }^{1}$; C. K. O. SILVA²; M.M.G.R. VIANNA², \\ O. CHIAVONE-FILHO ${ }^{1}$, C.A.O. NASCIMENTO ${ }^{2}$
}

${ }^{1}$ Universidade Federal do Rio Grande do Norte, Departamento de Engenharia Química

${ }^{2}$ Universidade de São Paulo, Departamento de Engenharia Química

E-mail para contato: silviashelly@hotmail.com

\begin{abstract}
RESUMO - A crescente preocupação com o meio ambiente vem viabilizando estudos que possibilitam a degradação de poluentes nocivos como os hidrocarbonetos policíclicos aromáticos (HPA). O Processo Oxidativo Avançado (POA), técnica baseada na produção de radicais, espécies capazes de reagir com vários compostos orgânicos, vem sendo utilizada atualmente. Os objetivos deste estudo foram avaliar a viabilidade de utilização do persulfato de sódio para degradar o fenantreno, utilizando um catalisador, a base de diatomita e ferro; investigar o melhor método de síntese para esses catalisadores e caracterizá-los. Os resultados da caracterização mostraram que os materiais são amorfos e a concentração de ferro foi $4 \%, 17 \%$ e $25 \%$ para diatomita bruta, CAT-5 e CAT-20, respectivamente. Os resultados mostraram que os materiais modificados apresentaram significativa atividade catalítica para ativar o persulfato de sódio e degradar o fenantreno, sendo a CAT-5 a mais reativa, degradando $56 \%$ de fenantreno em 72 horas de reação.
\end{abstract}

\section{INTRODUÇÃO}

De acordo com a agência ambiental americana United States Environmental Protection Agency (USEPA) foi publicada uma lista com 126 poluentes prioritários (PP), que abrangem pesticidas, bifenilas policloradas (PCB), metais pesados, hidrocarbonetos aromáticos e policíclicos aromáticos. Para que pertençam a essa lista é necessário que eles apresentem os seguintes comportamentos: serem carcinogênicos, mutagênicos, teratogênicos, tóxicos ao homem, serem persistentes e biocumulativos no meio ambiente. Fazem parte dessa lista 16 hidrocarbonetos policíclicos aromáticos (HPA), entre eles o fenantreno. A USEPA regulamenta e dispõe de métodos analíticos para identificar e quantificar esses poluentes (USEPA, 2012). 
O contaminante modelo usado para estudos envolvendo a interação de HPA no meio ambiente, o fenantreno $\left(\mathrm{C}_{14} \mathrm{H}_{10}\right)$, HPA formado por 3 anéis aromáticos, é frequentemente utilizado por ser considerado não carcinogênico para seres humanos.

Os tratamentos de solo contaminados com HPA pelos processos incineração e escavação são restritos e apresentam custo elevado. Os tratamentos do solo in situ, com estes compostos, são frequentemente mais utilizados por apresentarem melhor custo-benefício do que a remediação ex situ, (Palmroth et al, 2006).

Dentre as técnicas de tratamento in situ, tem-se a oxidação química in situ (In Situ Chemical Oxidation - ISCO), que é uma técnica viável para a remediação de aquíferos, sedimentos e solos contaminados com poluentes orgânicos. ISCO envolve a introdução de um oxidante químico no subsolo com a finalidade de transformar os contaminantes nas águas subterrâneas e/ou no solo em espécies químicas menos nocivas, podendo estas espécies chegar a mineralização completa (Huling e Pivetz, 2006).

$\mathrm{O}$ persulfato $\left(\mathrm{S}_{2} \mathrm{O}_{8}{ }^{-2}\right)$ é uma forma relativamente nova de oxidante que tem sido investigada, principalmente em escala de bancada. O oxidante pode ser ativado por diversos métodos para formar o forte radical sulfato $\left(\mathrm{SO}_{4}^{-\bullet}\right)$, esse tem potencial redox de $2,6 \mathrm{~V}$, assim sendo mais alto do que o potencial do íon persulfato $(2,1 \mathrm{~V})$, com isso a reatividade do radical será maior. A ativação desse oxidante pode se dá através das seguintes fontes: calor, luz UV, meio alcalino, ferro quelado, ozônio e peróxido de hidrogênio.

A partir da ativação por metais de transição (representado por Mt), um mol de persulfato produz 1 mol de radical, equação 1 .

$$
\mathrm{S}_{2} \mathrm{O}_{8}{ }^{2-}+\mathrm{Mt} \rightarrow \mathrm{Mt}^{+}+\mathrm{SO}_{4}{ }^{2-}+\mathrm{SO}_{4}^{-}
$$

A reação convencional de ativação do persulfato de sódio se dá a partir da ativação por $\mathrm{Fe}^{2+}$, para que os radicais sejam gerados é necessário um $\mathrm{pH}$ ácido. Este baixo $\mathrm{pH}$ pode afetar negativamente os sistemas naturais do solo e alterar suas características (Jorfi, 2013). Com isso, a necessidade de trabalhar com um $\mathrm{pH}$ baixo pode ser considerada como uma das desvantagens do método convencional para a ativação utilizando $\mathrm{Fe}^{2+}$.

Este trabalho tem como objetivo propor como tratamento do fenatreno, um método de ativação do persulfato a partir de um catalisador composto por um material suporte de baixo custo, a diatomita, sobre a qual é precipitada uma combinação de íons ferroso $\left(\mathrm{Fe}^{2+}\right)$ e férrico $\left(\mathrm{Fe}^{3+}\right)$ na forma de hidróxidos de ferro.

\section{METODOLOGIA}

\subsection{Síntese e Caracterização dos Materiais}

Os métodos de síntese de 2 catalisadores estão apresentados a seguir:

- $\quad$ CAT-20 - A obtenção do catalisador deu-se da seguinte maneira: utilizando-se $670 \mathrm{~g}$ de diatomita e adicionando-se lentamente a $754 \mathrm{~mL}$ de uma mistura de soluções aquosas contendo: $\mathrm{FeCl}_{3} \cdot 6 \mathrm{H}_{2} \mathrm{O}$ na concentração de 366 g.L.-1 e $\mathrm{FeSO}_{4} \cdot 7 \mathrm{H}_{2} \mathrm{O}$ na concentração de 183 g. $\mathrm{L}^{-1}$, em um reator de mistura intensa. A essa suspensão foi adicionada uma solução de $\mathrm{NaOH}\left(3,7 \mathrm{~L}, 20\right.$ mol. $\left.\mathrm{L}^{-1}\right)$ para a precipitação de óxidos de ferro. Após $24 \mathrm{~h}$ da adição da 
solução de $\mathrm{NaOH}$, o catalisador foi purificado inúmeras vezes por diálise, sob agitação magnética em placa, e seco em estufa a $60{ }^{\circ} \mathrm{C}$. Em seguida, o catalisador foi moído (desagregado), com almofariz e pistilo e depois peneirado e armazenado em frascos de vidro.

- $\quad$ CAT- 5 - A obtenção do catalisador deu-se da seguinte maneira: utilizando-se $100 \mathrm{~g}$ de diatomita adicionada lentamente a $112 \mathrm{~mL}$ de uma mistura de soluções aquosas contendo: sulfato férrico na concentração de 366 g.L $\mathrm{L}^{-1}$ e $\mathrm{FeSO}_{4} .7 \mathrm{H}_{2} \mathrm{O}$ na concentração de 183 g.L. ${ }^{-1}$, em um reator de mistura intensa. A essa suspensão foi adicionada uma solução de $\mathrm{NaOH}$ (220 $\left.\mathrm{mL}, 5 \mathrm{~mol} . \mathrm{L}^{-1}\right)$. A mistura foi homogeneizada por 1 hora. Após, o material obtido foi separado em duas partes: a primeira foi colocada diretamente na estufa a $60{ }^{\circ} \mathrm{C}$, sem lavagem (CAT$5 \mathrm{~N})$ e a segunda parte foi lavada com filtração a vácuo e colocada em estufa também a $60^{\circ} \mathrm{C}$. Após a secagem, o catalisador foi moído (desagregado), com almofariz e pistilo e depois peneirado e armazenado em frascos de vidro. O catalisador CAT-5N foi sintetizado da mesma forma que o CAT-5, entretanto não passou pelo processo de lavagem.

Para a obtenção do CAT-5 foi utilizada uma menor quantidade de água para lavagem e menor concentração da solução de hidróxido de sódio $\left(5 \mathrm{~mol} . \mathrm{L}^{-1}\right)$ do que no CAT-20 que foi preparado com hidróxido de sódio a $20 \mathrm{~mol}^{-1} \mathrm{~L}^{-1}$ e lavagem por diálise exaustiva. $\mathrm{O}$ primeiro catalisador sintetizado nesta pesquisa foi o CAT-20.

As técnicas utilizadas para a caracterização dos materiais foram: determinação da área superficial específica (ASE), difração de raios-x e fluorescência de raios-x.

\subsection{Tratamento de Fenantreno em Matriz Sólida}

Em cada reator (erlenmeyer de $125 \mathrm{~mL}$ ), pesou-se 15 gramas da areia contaminada com $200 \mathrm{mg}$ de fenantreno por kg de solo, sendo adicionados os volumes das soluções dos oxidantes e completados com água milli-Q até o volume de $30 \mathrm{~mL}$.

Nas reações com o catalisador, a quantidade de catalisador foi adicionada no frasco e homogeneizado, e o volume final de solução aquosa também foi mantido em $30 \mathrm{~mL}$. Os frascos foram acondicionados em banho termostático a uma temperatura de $32{ }^{\circ} \mathrm{C}$. Os ensaios controles (sem adição de oxidante) foram realizados em todas as reações, nas mesmas condições de tratamento. Os experimentos foram realizados em duplicata, e o erro experimental nesse estudo não foi maior do que $5 \%$.

Ensaios com os catalisadores : Realizaram-se testes em batelada, comparando os diferentes catalisadores preparados, CAT-20, CAT-5 e CAT-5N (catalisador não lavado). Em cada reator (erlenmeyer) adicionou-se 2,5 gramas do respectivo catalisador com persulfato de sódio $\left(25 \mathrm{~g} \mathrm{~L}^{-1}\right)$. O tempo de remediação desses ensaios foi mantido constante em 72 horas.

Teste ativação convencional e ativação promovida pela diatomita modificada: Realizaram-se testes com os oxidantes persulfato de sódio e peróxido de hidrogênio, ativados via $\mathrm{Fe}^{2+}$ e comparados com a ativação promovida pelo CAT-5, sendo mantido constante o tempo de remediação em 72 horas. As condições de oxidantes, $\mathrm{Fe}^{2+}$ e quantidade de CAT-5 estão apresentadas na Tabela 1. Os ensaios foram realizados sem ajuste de $\mathrm{pH}$. 
Tabela 1 - Concentrações de oxidantes, sulfato ferroso e catalisador

\begin{tabular}{|c|c|c|c|c|}
\hline Oxidantes & $\begin{array}{c}\text { Concentração } \\
\text { oxidante } \\
(\text { mol.L-1) }\end{array}$ & $\begin{array}{c}\text { Concentração } \\
\text { sulfato ferroso } \\
\left(\text { mol.L }^{-1}\right)\end{array}$ & $\begin{array}{l}\text { Catalisador } \\
\text { (gramas)* }^{*}\end{array}$ & $\mathrm{pH}_{\text {inicial }}$ \\
\hline Persulfato de sódio & $0,1 * *$ & 0,003 & & 3 \\
\hline $\begin{array}{l}\text { Persulfato de sódio }+ \\
\text { CAT-5 }\end{array}$ & 0,1 & & 2,5 & 11 \\
\hline
\end{tabular}

\section{EXTRAÇÃO E ANÁLISE}

Para quantificação da contaminação residual e dos possíveis sub-produtos formados durante a oxidação, alíquotas de 2,5 gramas de areia foram retiradas de cada reator, sendo cada alíquota adicionada em vial de $40 \mathrm{~mL}$, juntamente com $10 \mathrm{~mL}$ de solvente hexano:cetona (1:1). A homogeneização, amostra-solvente, foi realizada utilizando vortex por 5 segundos. Em seguida, cada vial com amostra e solvente foi submetido a ultrassom durante 30 minutos para extração sólido-liquido. Após a extração, cada amostra foi passada pelo vortex novamente por 5 segundos. $\mathrm{O}$ extrato foi filtrado, por filtração comum, contendo sulfato de sódio no papel de filtro. Esse extrato filtrado foi então colocado em vial de $1,5 \mathrm{~mL}$ para injeção em um Cromatógrafo Gasoso acoplado ao Espectrômetro de Massa (CG-EM), modelo GC-17A da Shimadzu. Os métodos analíticos, extração e análise, foram empregados de acordo com métodos USEPA.

\section{RESULTADOS}

\subsection{Caracterização}

As áreas superficiais específicas (ASE) da diatomita bruta, CAT-5 e CAT-20 foram de $116 \mathrm{~m}^{2} \cdot \mathrm{g}^{-1}, 128 \mathrm{~m}^{2} \cdot \mathrm{g}^{-1}$ e $191 \mathrm{~m}^{2} \cdot \mathrm{g}^{-1}$, respectivamente. Com o aumento da área superficial específica dos materiais gera-se um aumento de reatividade, portanto quanto maior a ASE, maior a reatividade (capacidade de participar de reações químicas) da partícula.

Com base nas análises de fluorescência de raios-X para as amostras: diatomita bruta, CAT-5 e CAT-20, que apresentaram os seguintes teores de ferro total: 4,7\%, 17,6\% e $25,6 \%$, respectivamente. Como previsto, os teores de ferro obtidos nas amostras indicam um significativo aumento na quantidade de ferro após a síntese, este presente na superfície das amostras é a espécie responsável pela ativação dos oxidantes. 


\subsection{Teste dos Catalisadores}

Com o objetivo de avaliar a eficiência dos novos materiais preparados (CAT-5 e CAT$5 \mathrm{~N}$ ), foram realizados testes de degradação para comparar com o CAT-20, cujos resultados estão apresentados na Figura 1. No ensaio com CAT-20 a degradação foi $46 \%$ de fenantreno. Já, no ensaio com CAT-5, a degradação desse contaminante foi de $56 \%$. Com base nesses resultados, verificou-se que as alterações realizadas no CAT-5 foram mais eficientes na degradação do contaminante.

Figura 1 - Resultado de degradação do fenantreno $\left(200 \mathrm{mg} \mathrm{kg}^{-1}\right)$ obtida em 72 horas comparando os catalisadores sintetizados. Persulfato de sódio a $0,1 \mathrm{~mol} \mathrm{~L}^{-1}\left(25 \mathrm{~g} \mathrm{~L}^{-1}\right)$ e $2,5 \mathrm{~g}$ de catalisador. Temperatura a $32^{\circ} \mathrm{C}$.

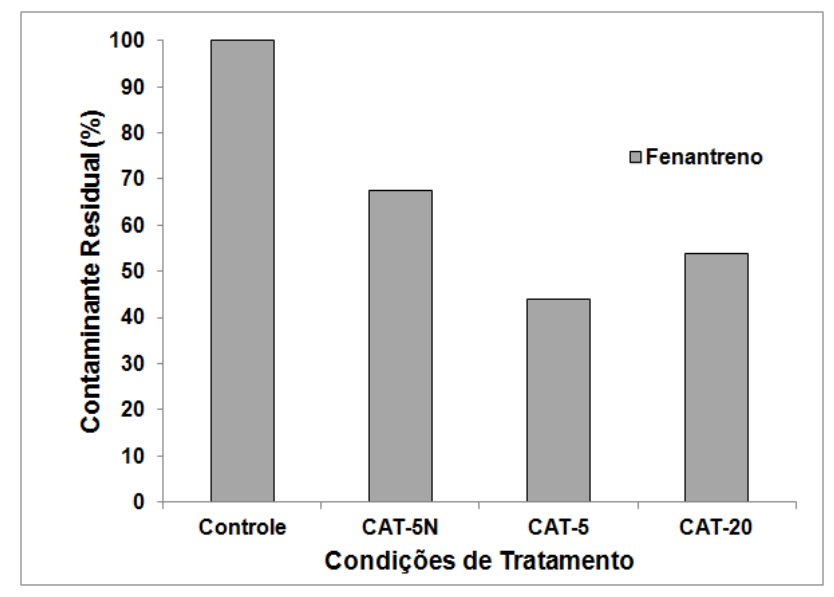

O catalisador que se obteve a menor degradação dos contaminantes foi o CAT-5N, possibilitando-se observar que a etapa de lavagem é necessária na síntese do catalisador, em virtude de nessa etapa possivelmente ocorrer a remoção do excesso de $\mathrm{NaOH}$ e outros subprodutos indesejáveis que podem ser formados durante a síntese.

O CAT-5 foi selecionado para os estudos posteriores, baseando-se nos resultados obtidos, já que este apresentou uma melhor eficácia na degradação dos contaminantes, e para sua síntese foi utilizado uma menor quantidade de água e menor concentração de hidróxido de sódio. Portanto, pode-se considerar que o CAT-5 é mais viável, ambientalmente e economicamente, do que o CAT-20.

Os resultados apresentados na Figura 2 são referentes ao controle sem adição de oxidante, com e sem CAT-5. O resultado do ensaio Controle CAT-5 (ensaio com catalisador sem adição de oxidante) mostra que o catalisador sintetizado apresenta propriedade de adsorção do fenantreno, visto que, ocorreu uma redução da concentração desse contaminante em 15\% em relação ao ensaio controle sem CAT-5, em 72 horas de remediação.

Na Figura 2 observaram-se também os resultados dos testes com o oxidante persulfato de sódio ativado via $\mathrm{Fe}^{2+}$ e pelo CAT-5, em 72 horas de reação. Utilizando o oxidante ativado, os percentuais de degradação do fenantreno foram $20 \%$ (via Fe ${ }^{2+}$ ) e $56 \%$ (CAT-5). 


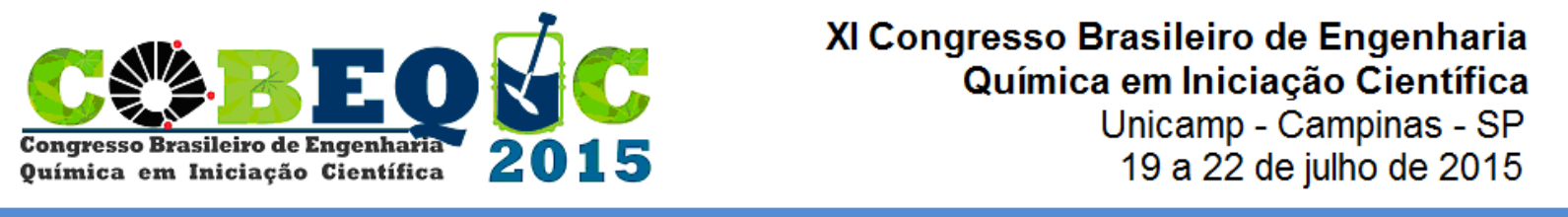

Figura 2 - Resultado de degradação do fenantreno $\left(200 \mathrm{mg} \mathrm{kg}^{-1}\right)$ comparando métodos de ativação do persulfato de sódio via $\mathrm{Fe}^{2+}$ e por CAT-5. Persulfato de sódio a $0,1 \mathrm{~mol} \mathrm{~L}^{-1}(25 \mathrm{~g}$ $\left.\mathrm{L}^{-1}\right)$ e $2,5 \mathrm{~g}$ de catalisador, 72 horas de remediação e temperatura de $32^{\circ} \mathrm{C}$.

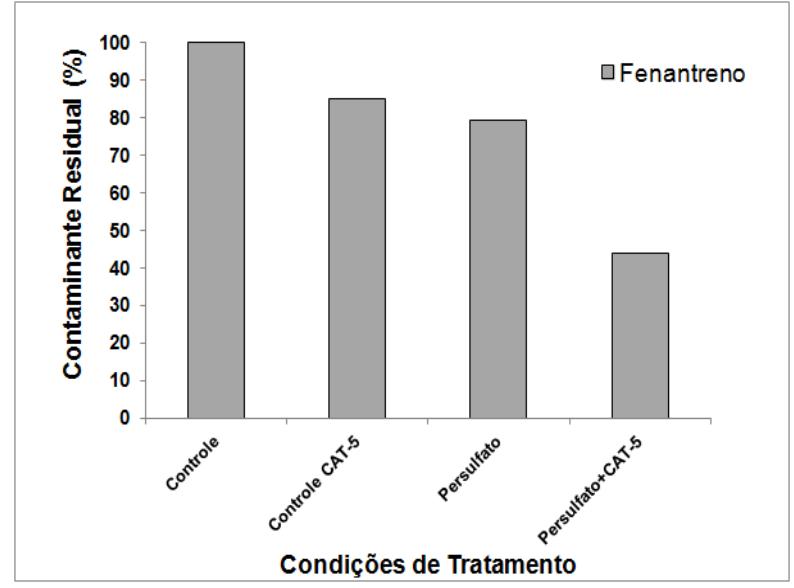

\section{CONCLUSÃO}

Com base nas análises de área superficial específica notou-se um aumento de área, que implica em uma maior reatividade, pois quanto maior a ASE, maior a reatividade (capacidade de participar de reações químicas) da partícula. Os resultados de fluorescência de raios-X indicou um aumento substancial do teor de ferro após a modificação

A partir dos ensaios realizados pode-se observar que dos catalisadores sintetizados, CAT-20, CAT-5, CAT-5N (não lavado), o material que apresentou um melhor desempenho tanto econômico quanto ambiental foi o CAT-5.

\section{REFÊNCIAS}

JORFI, S.; REZAEE, A.; MOHEB-ALI, G.; JAAFARZADEH, N. Pyrene removal from contaminated soils by modified Fenton oxidation using iron nano particles. Journal of Environmental Health and Science Engineering, 1, 2013, 11-17.

HULING, S.; PIVETZ, B. Engineering Issue: In Situ Chemical Oxidation, USEPA 600/R-06/072. U.S. EPA, Office of Research and Development, 2006.

PALMROTH, M. R. T.; LANGWALDT, J.H.; AUNOLA, T.A.; GOI, A.; MÜNSTER, U. PUHAKKA, J.A.; TUHKANEN, T.A. Effect of modified Fenton's reaction on microbial activity and removal of PAHs in creosote oil contaminated soil. Biodegradation, 2006.

United States Environmental Protection Agency - USEPA, 2012. Disponível em: $<$ http://water.epa.gov/scitech/methods/cwa/pollutants-background.cfm $>$. Acesso em 4 ago 2014. 